\title{
Two-Stage Over-the-Air (OTA) Test Method for LTE MIMO Device Performance Evaluation
}

\author{
Ya Jing, Xu Zhao, Hongwei Kong, Steve Duffy, and Moray Rumney \\ Agilent Technologies, Santa Clara, CA 95051, USA \\ Correspondence should be addressed to Moray Rumney, moray_rumney@agilent.com \\ Received 11 February 2012; Accepted 28 March 2012 \\ Academic Editor: Ryan J. Pirkl \\ Copyright () 2012 Ya Jing et al. This is an open access article distributed under the Creative Commons Attribution License, which \\ permits unrestricted use, distribution, and reproduction in any medium, provided the original work is properly cited.
}

With MIMO technology being adopted by the wireless communication standards LTE and HSPA+, MIMO OTA research has attracted wide interest from both industry and academia. Parallel studies are underway in COST2100, CTIA, and 3GPP RAN WG4. The major test challenge for MIMO OTA is how to create a repeatable scenario which accurately reflects the MIMO antenna radiation performance in a realistic wireless propagation environment. Different MIMO OTA methods differ in the way to reproduce a specified MIMO channel model. This paper introduces a novel, flexible, and cost-effective method for measuring MIMO OTA using a two-stage approach. In the first stage, the antenna pattern is measured in an anechoic chamber using a nonintrusive approach, that is without cabled connections or modifying the device. In the second stage, the antenna pattern is convolved with the chosen channel model in a channel emulator to measure throughput using a cabled connection.

\section{Introduction}

OTA test has been standardized by CTIA and 3GPP to evaluate the end-to-end performance of SISO devices. Research results show that field performance of MIMO devices is more complex and depends on a number of factors, including self-desensitization, baseband processing, the propagation conditions, and the antenna performance. Of these, the propagation conditions and the antenna performance are paramount. With MIMO technology being adopted by the wireless communication standards LTE and HSPA+, cellular operators are pushing for the introduction of MIMO OTA performance requirements. A study of MIMO OTA for HSPA+ and LTE was proposed to 3GPP in [1]. Since then, MIMO OTA research has attracted wide interest from both industry and academia. Parallel studies are underway in COST2100 [2], CTIA [3], and 3GPP RAN WG4 [4]. 3GPP RAN WG4 has conducted a MIMO OTA measurement campaign using a common device pool. Some Agilent results in this paper come from the measurement campaign [5]. Due to the complexity of the problem, after about three-year study and discussion, there are still many issues unsolved, and further research work is required.

Unlike SISO, MIMO technology is designed to exploit a rich multipath channel. For MIMO OTA performance evaluation, it is thus necessary to introduce a realistic MIMO channel model. The major challenge for testing MIMO OTA is how to emulate an RF environment which accurately reflects the MIMO device's performance in the real wireless propagation environment. The objective of the test is to get comparable performance between the field and the lab. Several methods for performing MIMO OTA test have been proposed to 3GPP RAN WG4, COST2100, and CTIA. These methods fall into four major categories [6]: the reverberation chamber, the two-stage method, the multiprobe anechoic chamber, and the two channel anechoic chamber. In this paper, we will introduce the two-stage MIMO OTA method and provide comparative measurement results with other methods. The paper is organized as follows. Section 2 provides a brief introduction of the two-stage MIMO OTA methodology and experimental procedure. In Section 3, the test results of the two-stage method using nonintrusive antenna pattern and throughput measurements are given. Section 4 provides a summary and some conclusions.

\section{Two-Stage MIMO OTA Method}

2.1. Two-Stage Method Description. The concept of the two-stage method is shown in Figure 1. The thorough 

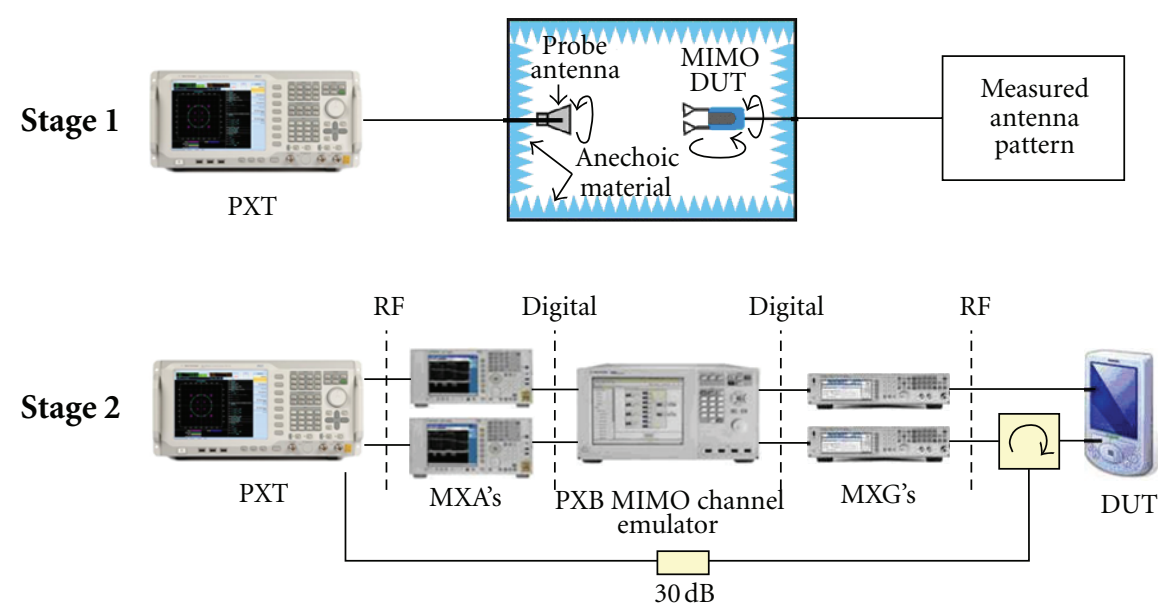

Figure 1: Concept of the two-stage method.

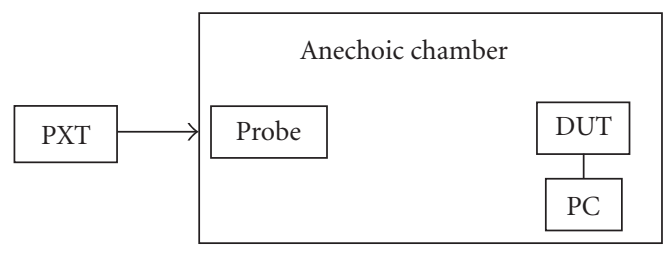

Figure 2: First-stage antenna pattern measurement.

introduction and analysis for this method can be found in [7]. This method divides the MIMO OTA test into two stages:

(1) measuring the device antenna pattern inside an anechoic chamber;

(2) using a commercial MIMO channel emulator, convolve the measured antenna pattern with the desired channel model to provide the stimulus for a conducted throughput test on the DUT.

The method is designed to provide a cost-effective MIMO OTA test solution by reusing the existing SISO OTA anechoic chamber for radiated antenna pattern measurements and then using a MIMO channel emulator to evaluate the DUTs throughput performance. [8, 9] give the power and relative phase definition for MIMO antenna pattern measurement. The chamber for antenna pattern measurement is set up as described in 3GPP TS 34.114 [10], where the DUT is put into a chamber, and each antenna element's far zone pattern is measured. The influence of human body loss can be measured by attaching the DUT to a SAM head and/or hand when doing the antenna pattern measurements. The MIMO antenna pattern and the desired multipath channel model are then convolved using a MIMO channel emulator like the Agilent N5106A to emulate the integrated channel. [11] explains how to apply measured antenna pattern to ray-based and correlation-based channel models for OTA test and also validates that combining with these two different models can get equivalent effects by simulation results. To measure the DUT performance through this integrated channel, the base station (BS) emulator is connected to the MIMO channel emulator and then to the MIMO DUT's temporary antenna ports via approved RF cables. These DUT ports are the standard ones provided for conducted conformance tests. By controlling the power settings of the channel emulator and also the integrated channel, the end-to-end throughput with the MIMO antenna radiation influence can be measured. [5, 12,13 ] provide detailed information on experiment setup and some LTE devices' performance results tested by this method. In [13], the capacity simulation results based on the measured antenna pattern are also given, and the test results show that the two-stage method throughput test results rank the devices consistently as compared with the capacity simulation results.

There are several advantages to this two-stage test method: the method can reuse existing SISO OTA anechoic chambers to make the antenna pattern measurements; only two-channel emulator outputs are required (to match the number of device receiver inputs) regardless of the complexity of the chosen channel model, the method is consequently easily scalable to higher order MIMO due to the reduced number of instruments required compared to some multiprobe methods; the channel models are highly accurate due to being implemented electronically and are also fully flexible and can be altered to suit any desired operating conditions such as indoor-outdoor, high or low Doppler spread, high or low delay spread, beam width, in $2 \mathrm{D}$ or full $3 \mathrm{D}$, and so forth.

In order to accurately measure the antenna pattern of the intact device, the DUT needs to support amplitude and relative phase measurements of the antennas. This is not currently a standardized feature of devices although the concept has been prototyped and in principle is not dissimilar to other test features that have been standardized for other conformance tests such as loopback to enable receiver BER measurements. There is also further research being carried out to extend the first-stage pattern measurement to take account of DUT self-desensitization which otherwise is not measured. 


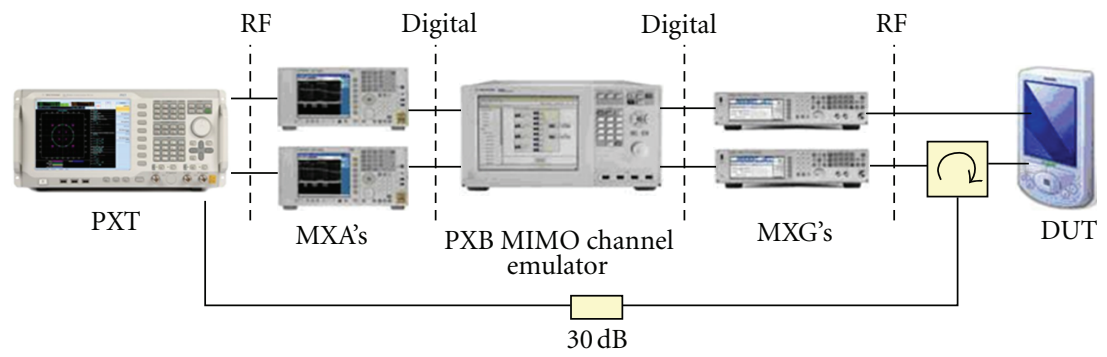

Figure 3: Second-stage conducted throughput measurement.
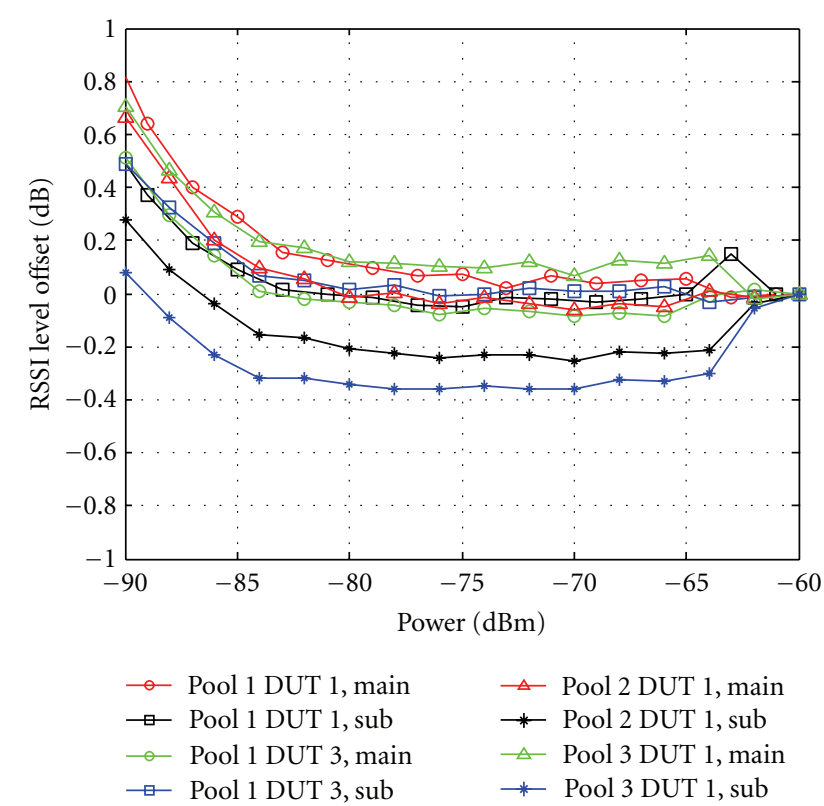

FIgURE 4: RSSI linearity measurement for DUTs from Company A.

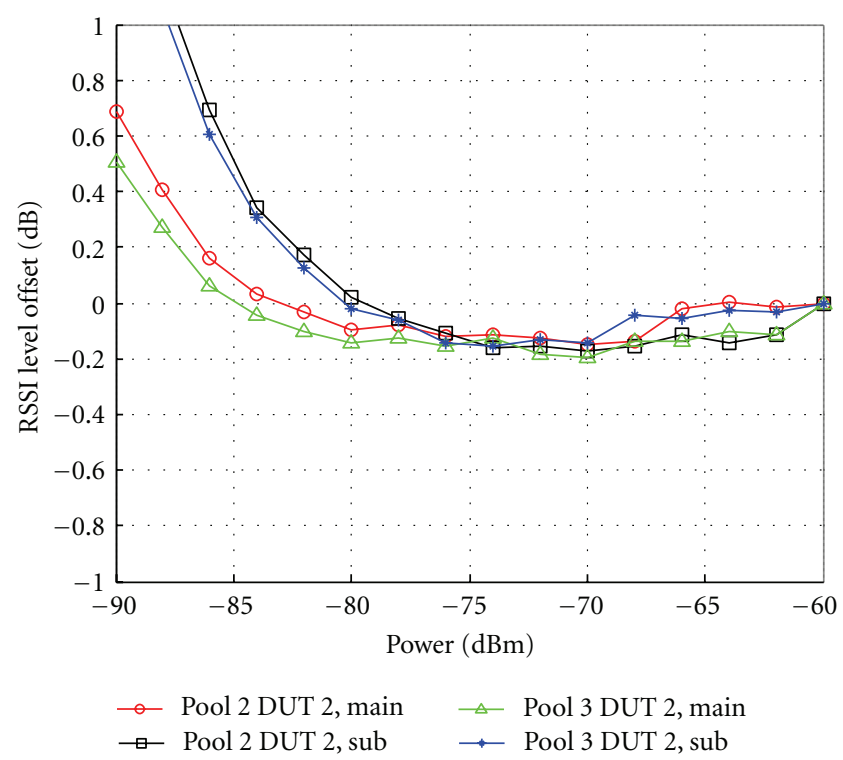

FIGURE 5: RSSI linearity measurement for DUTs from Company B.

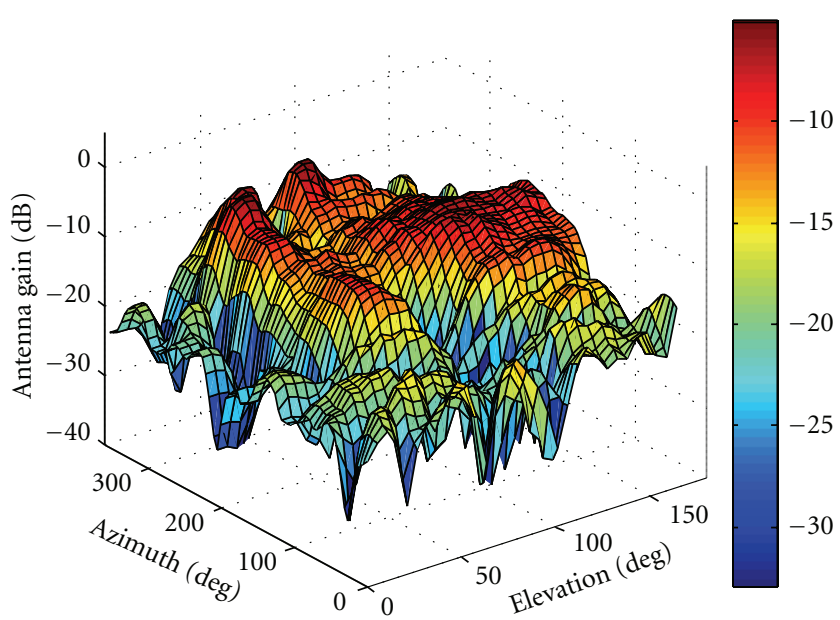

FIgURE 6: Pool 1 DUT 3 main antenna pattern on polarization V.

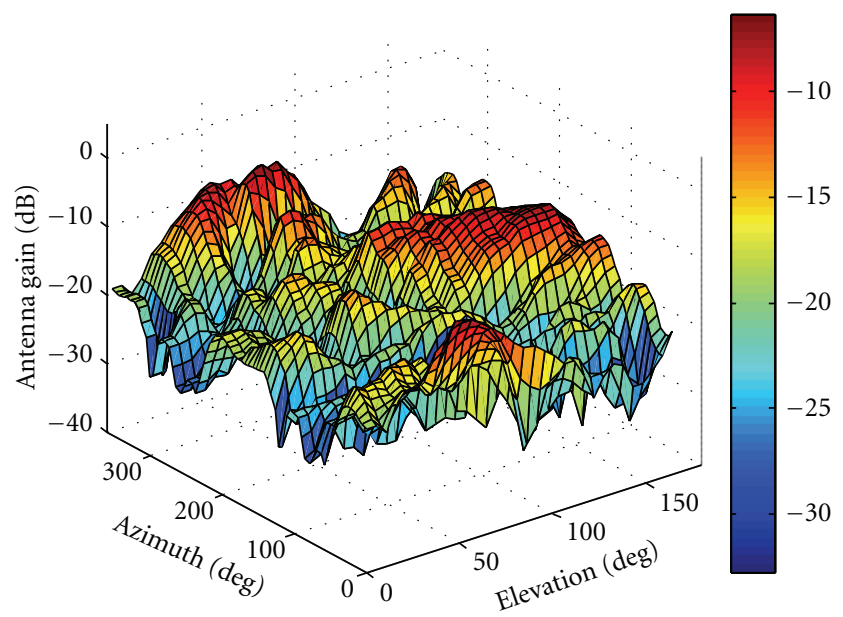

Figure 7: Pool 1 DUT 3 main antenna pattern on polarization $H$.

\subsection{Experimental Setup and Procedure}

2.2.1. Active Pattern Measurement Setup. Currently some LTE MIMO devices support a nonintrusive antenna pattern measurement function, which means that DUT can report amplitude, and relative phase measurements of the antennas $[14,15]$ validate the reported amplitude and phase's accuracy by designed experiments. 


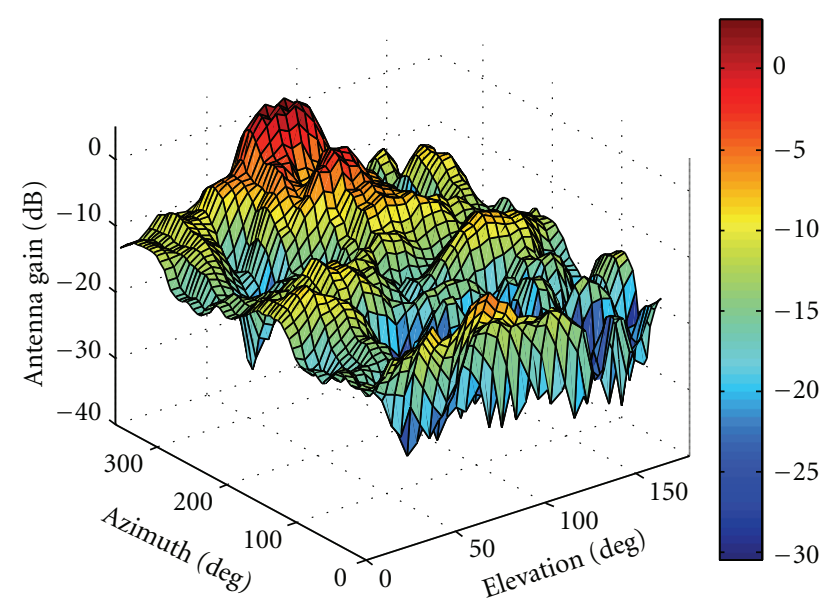

FIgURE 8: Pool 1 DUT 3 subantenna pattern on polarization V.

We have done active 3D antenna pattern measurement on some devices in a SATIMO anechoic chamber. The antenna pattern measurement setup is shown in Figure 2. More information on these LTE devices and test setup can be found in [5]. There is software running on the laptop which controls the DUT to log the received power and phase per antenna. Before the antenna pattern measurement is carried out, the path loss between the reference antenna and DUT was calibrated to obtain the absolute power incident at the DUT. Agilent and SATIMO jointly defined a 3D antenna pattern measurement process. By using this process, the probe position and table rotation information can be correlated with the power and phase measurements in the UE log file. Agilent also developed a Matlab parser, which can extract the 3D antenna pattern data, and perform path loss, probe, and received signal strength indicator (RSSI) linearity calibration automatically from the logged file.

2.2.2. Pattern Power Linearity Calibration. Because the UE power measurement may not be completely linear, a further linearity calibration was performed using a calibrated signal in conducted mode. Using this correction, the antenna pattern was calibrated according to the measured power linearity curves. Section 3 will show some measured power linearity curves.

2.2.3. Throughput Test Setup. The throughput test configuration is shown in Figure 3. The Agilent E6621A PXT is used as the LTE BS emulator, the Agilent N5106A PXB is used as the channel emulator, and the Agilent MXG and MAX is used for RF upconversion and downconversion. The DUT has two antennas: the first antenna is the main antenna which acts as both transmitter and receiver. The second antenna is the supplementary antenna which acts only as a receiver. An RF circulator is used to separate the uplink and downlink of the main antenna. A $30 \mathrm{~dB}$ attenuator is used in the uplink to avoid interference between the uplink and the downlink at the BS emulator. The test setup is very similar to a typical cable conducted test for throughput

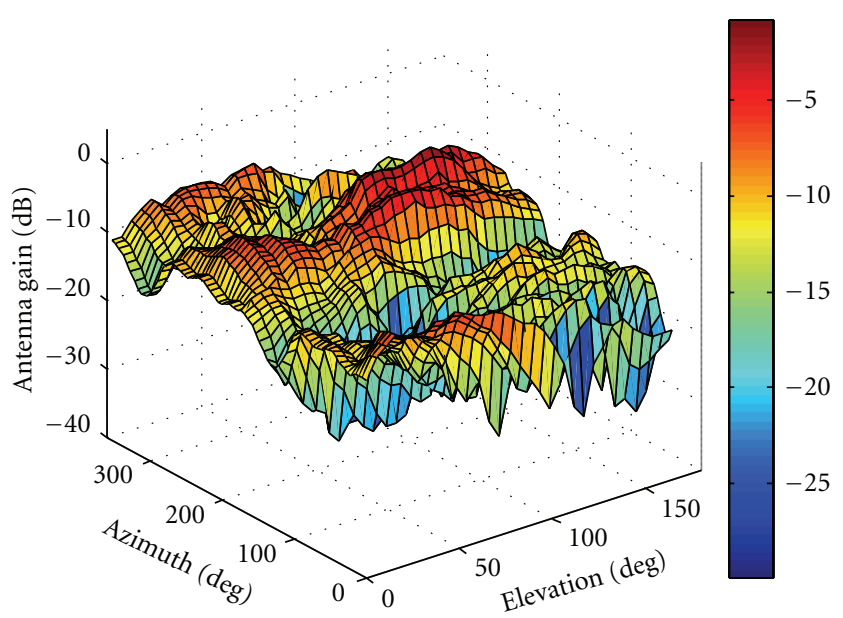

Figure 9: Pool 1 DUT 3 sub antenna pattern on polarization $\mathrm{H}$.

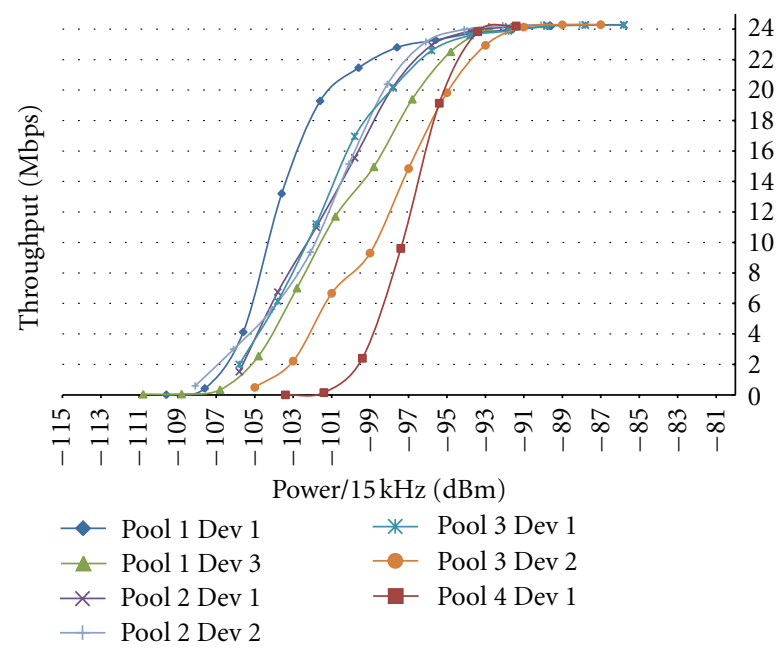

FIgURE 10: Throughput performance for single-cluster Umi channel model.

with fading. The difference is that in the MIMO OTA test, the measured antenna pattern of the DUT is loaded into the channel emulator and used together with the chosen MIMO channel model to emulate the influence of the DUT's antenna (correlation, power imbalance, and antenna gain) on the multipath fading. The detailed test procedure is referred to in Agilent proposal [12].

\section{Experimental Results}

This section gives the measurements results for Agilent's 3GPP measurements [5], including power linearity, 3D antenna pattern, and throughput. There is also other company which does the two-stage throughput test on same DUT. In the latest 3GPP MIMO OTA status report [16], it is summarized that under similar test conditions two companies get comparable results. [17] provides the throughput comparison with other methods. The comparison shows for some DUTs, the reverberation chamber method, 


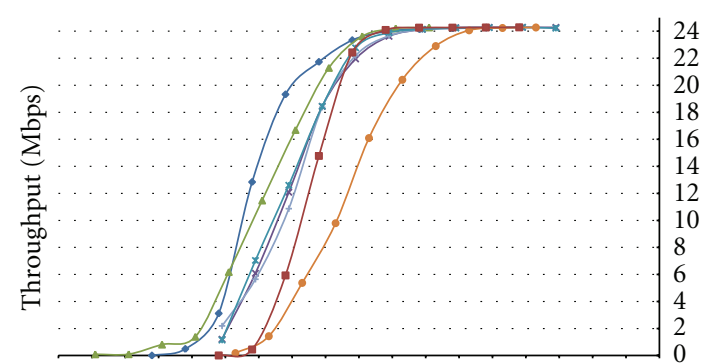

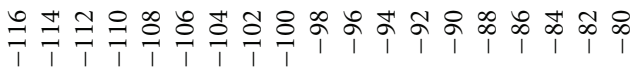

$$
\begin{array}{cc}
\multicolumn{2}{c}{\text { Power/15 kHz }(\mathrm{dBm})} \\
\leftarrow \text { Pool } 1 \text { Dev } 1 & - \text { Pool } 3 \text { Dev } 1 \\
\leftarrow \text { Pool } 1 \text { Dev } 3 & - \text { Pool } 3 \text { Dev } 2 \\
\leftarrow \text { Pool } 2 \text { Dev } 1 & - \text { Pool } 4 \text { Dev } 1
\end{array}
$$

FIGURE 11: Throughput performance for multicluster Umi channel model.

the two-stage method, and the multi-probe anechoic chamber method can provide comparable results, but on some DUTs the results from different companies have big departures. The most key reason is that test environments from different companies are not completely same. Then, further investigation work is continued.

Power measurement linearity tests were performed on seven DUTs. In the experiment, we tune the signal power feed into DUTs from $60 \mathrm{dBm}$ to $-90 \mathrm{dBm}$ in $2 \mathrm{~dB}$ steps and record the measured RSSI in each step. The RSSI recorded at $-60 \mathrm{dBm}$ signal power is chosen as the reference to calculate the RSSI linearity at different power levels. Figures 4 and 5 show RSSI linearity results for six DUTs from two different companies. These figures demonstrate that the uncorrected UE power measurement linearity was generally very good, and all DUTs have no more than $1 \mathrm{~dB}$ offset over a $30 \mathrm{~dB}$ power range. However, any linearity error is calibrated out from the measured antenna pattern in chamber before the throughput test.

Seven DUTs' 3D antenna patterns were measured in the SATIMO anechoic chamber. To save space, only one DUT pattern is given as an example from Figures 6, 7, 8, and 9. These figures demonstrate that the measured pattern is very smooth over the whole sphere, and we also performed the other experiments which validate the pattern measurement repeatability.

Throughput tests were performed on seven DUTs. Once the $3 \mathrm{D}$ antenna pattern is known, it is very simple using the two-stage method to calculate the 2D performance for any elevation angle to emulate the results obtained from the 2D multi-probe method. Here, we only show the throughput results for an elevation angle of 90 degrees, which is parallel to the ground. For each power level, eight uniform distributed orientation points were tested, and the overall results were the average. Figures 10, 11, and 12 show the throughput measurement results for single-cluster Umi, multi-cluster Umi, and multi-cluster Uma channel models [18].

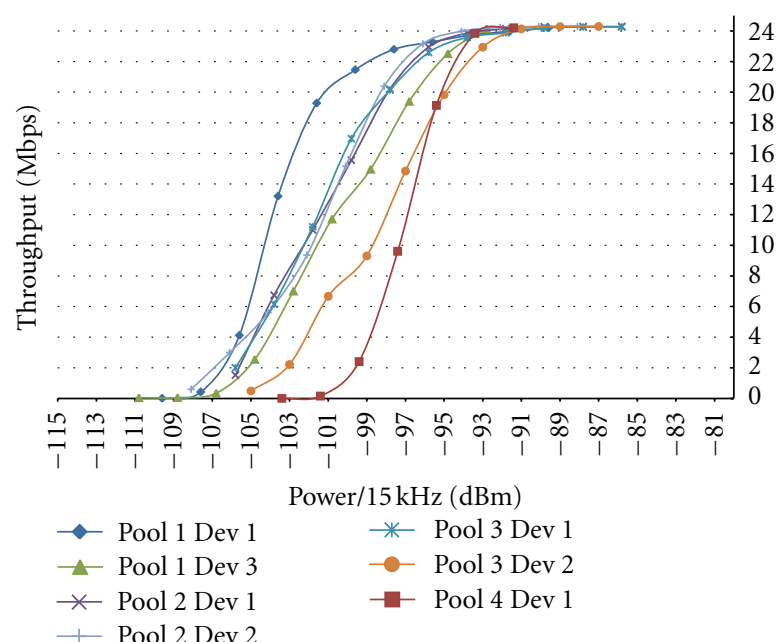

FIGURE 12: Throughput performance for multicluster Uma channel model.

These results show that:

(1) no matter which of the three channel models is used, Pool 1 DUT 1 is the best DUT;

(2) no matter which of the three channel models is used, Pool 2 DUT 1, Pool 2 DUT 2, and Poo 3 DUT 1 show very similar performance;

(3) because the eNB antenna was set to be uncorrelated, the performance for multi-cluster Umi and multicluster Uma are similar;

(4) the performance curves for the single-cluster channel models show wider spread than that for multi-cluster channel models, which demonstrates that a narrower angular spread makes the test more sensitive to DUT performance;

(5) Pool 1 DUT 3's ranking sequence shows significant change between the single-cluster model and multicluster models, which demonstrates that Pool 1 DUT 3 is more sensitive to channel power angular spread;

(6) Pool 4 DUT 1's ranking relative to Pool 3 DUT 2 changed when switching from single-cluster to multicluster, which also demonstrates Pool 4 DUT 1 is sensitive to channel power angular spread.

\section{Summary}

In this paper, the two-stage MIMO OTA test method is introduced. Using the antenna test mode of the DUT a nonintrusive $3 \mathrm{D}$ antenna pattern measurement is performed and verified by RSSI linearity calibration. The DUT's MIMO throughput is then measured using a conducted test by convolving the antenna pattern with the desired channel model. This approach enables the creation of arbitrarily complex $2 \mathrm{D}$ or $3 \mathrm{D}$ channel models including the antenna impact while only requiring a simple SISO anechoic chamber to measure the antenna pattern. The two-stage method can 
thus provide a low-cost and accurate method for evaluating end-to-end MIMO performance.

\section{Acknowledgments}

The authors would like to thank SATIMO which provided access to its anechoic chamber laboratory to perform the OTA measurements.

\section{References}

[1] Vodafone, "Study item proposal for MIMO OTA," 3GPP RAN4 R4-090903.

[2] COST2100, http://www.cost2100.org/.

[3] CTIA, http://www.ctia.org/.

[4] 3GPP, http://www.3gpp.org/.

[5] Agilent, "Results for two-stage LTE MIMO OTA Round Robin Testing," 3GPP R4-116046.

[6] TR.37.976, "Measurement of radiated performance for MIMO and multiple antenna reception for HSPA and LTE terminals".

[7] Agilent, "MIMO OTA test methodology proposal," 3GPP R4091361.

[8] Agilent, "Power and relative phase definition for antenna pattern measurement," 3GPP R4-115288.

[9] Agilent, "Definition of UE pattern measurement function based on RSRP definition," 3GPP R4-116105.

[10] 3GPP TS 34.114, User Equipment (UE)/Mobile Station (MS) Over The Air (OTA) Antenna Performance Conformance Testing.

[11] Agilent, "Applying measured MIMO antenna patterns to MIMO channel models for OTA analysis," 3GPP R4-091949.

[12] Agilent, "Preliminary LTE MIMO OTA test results using twostage method," 3GPP R4-112184.

[13] Agilent, "LTE MIMO OTA test results and analysis," R4114188.

[14] Qualcomm, "TP on including antenna pattern based test procedure for LTE MIMO OTA test plan,” 3GPP R4-104154.

[15] Agilent, "Experimental validation on handset antenna pattern measurement accuracy," 3GPP R4-111742.

[16] 3GPP RP-111541, "MIMO OTA status reports".

[17] Agilent, "Summary of two-stage measurement results," 3GPP R4-116104.

[18] 3GPP TR 25.996, Spatial channel model for MIMO simulation. 

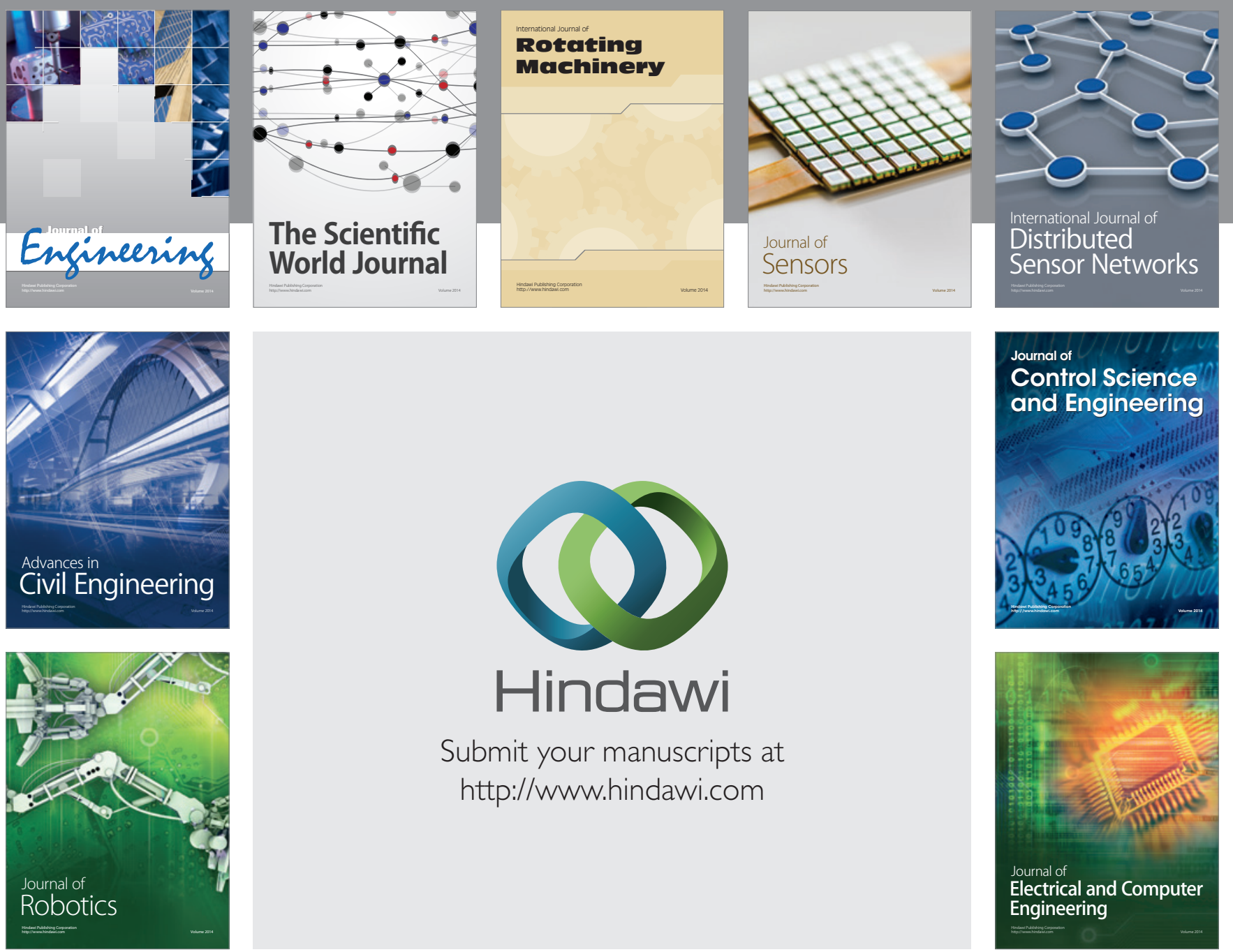

Submit your manuscripts at

http://www.hindawi.com
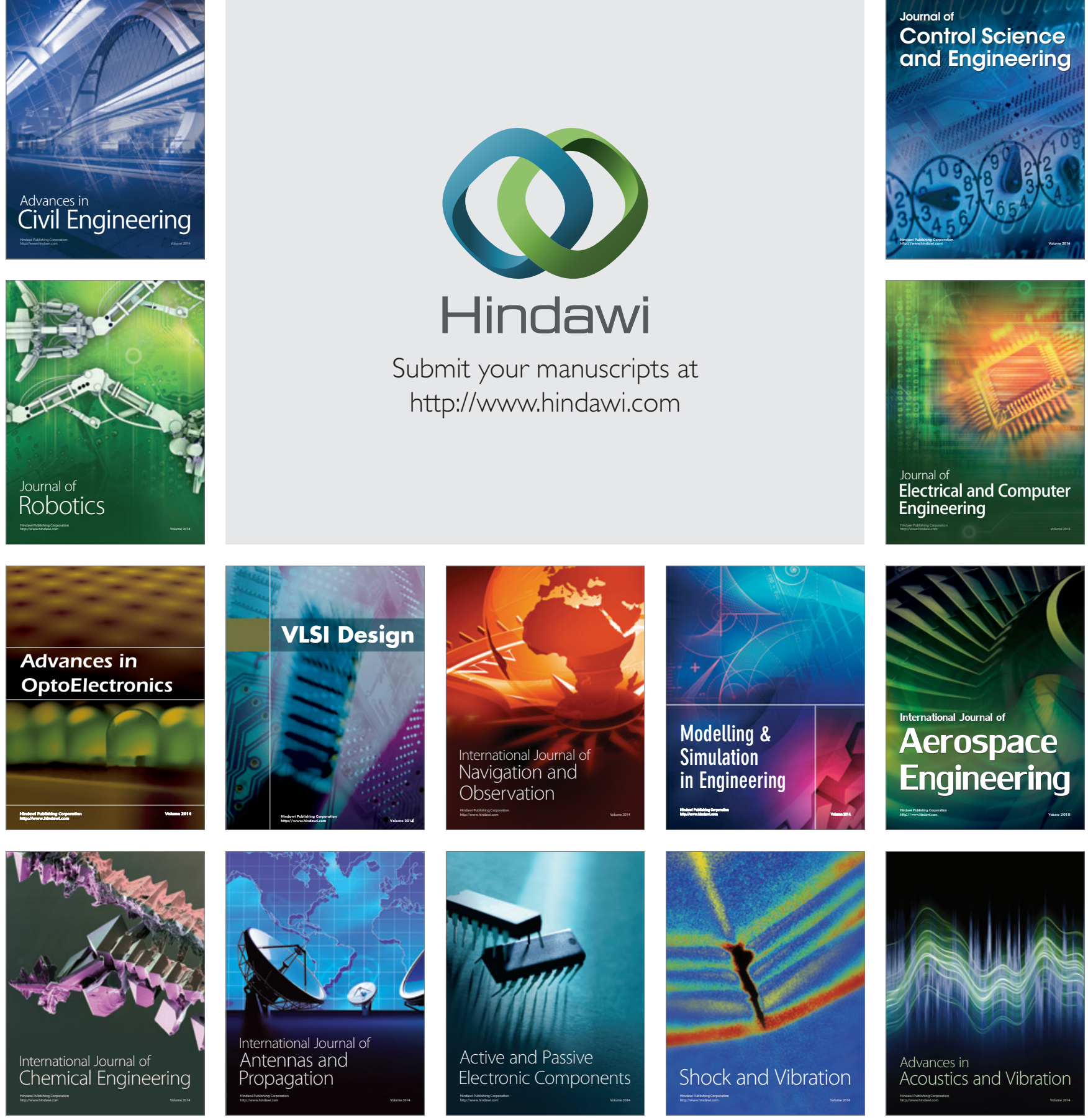